\title{
Primary adenovirus-specific cytotoxic T lymphocyte response occurs after viral clearance and liver enzyme elevation
}

\author{
J Chen ${ }^{1}$, AJ Zajac ${ }^{2}$, SA McPherson ${ }^{3}, \mathrm{H}-\mathrm{C} \mathrm{Hsu}{ }^{1}, \mathrm{P} \mathrm{Yang}^{1}, \mathrm{Q} \mathrm{Wu}^{1}, \mathrm{X} \mathrm{Xu}^{1}, \mathrm{X}$ Wang $^{1}, \mathrm{~K}$ Fujihashi ${ }^{4}$, \\ DT Curiel ${ }^{1,5,6}$ and JD Mountz ${ }^{1,7}$ \\ ${ }^{1}$ Department of Medicine, University of Alabama at Birmingham, Birmingham, AL, USA; ${ }^{2}$ Department of Microbiology, \\ University of Alabama at Birmingham, Birmingham, AL, USA; ${ }^{3}$ Center for AIDS Research, University of Alabama at Birmingham, \\ Birmingham, AL, USA; ${ }^{4}$ Department of Pediatric Dentistry, University of Alabama at Birmingham, Birmingham, AL, USA; \\ ${ }^{5}$ Department of Pathology and Surgery, University of Alabama at Birmingham, Birmingham, AL, USA; \\ ${ }^{6}$ Division of Human Gene Therapy and Gene Therapy Center, University of Alabama at Birmingham, Birmingham, AL, USA; and \\ ${ }^{7}$ Veteran Administration Medical Center, Birmingham, AL, USA
}

The virus-specific cytotoxic $T$ lymphocyte (CTL) response is a major obstacle to effective delivery of adenovirus gene therapy. However, its relative role in viral clearance, transgene elimination and hepatotoxicity remains unclear. In this paper, we present an analysis of viral clearance and liver toxicity in relation to the induction of the virus-specific CD8 T-cell response revealed by an MHC class I tetramer. A surprisingly high number of tetramer ${ }^{+} C D 8 T$ cells were found in the liver and lung and reached peak values at days 8 and 10 , respectively, post-infection. Nearly $100 \%$ of these tetramer ${ }^{+}$CD8 $T$ cells expressed high levels of granzyme $B$ and IFNy. Remarkably, liver viral load and liver enzyme elevation peaked early, at days 2 and 4, respectively, postinfection, before the specific CTL response was detectable. After generation of CTLs, there was only minimal liver damage or further decrease in virus titer. These results indicated that the primary peak response of tetramer ${ }^{+}$CTLs does not correlate with the elimination of adenovirus or liver cytotoxic response.

Gene Therapy (2005) 12, 1079-1088. doi:10.1038/

sj.gt.3302494; published online 7 April 2005

Keywords: adenovirus; cytotoxic T lymphocyte response; class I MHC tetramer; viral clearance; liver enzyme elevation

\section{Introduction}

Clearance of adenovirus (Ad) and subsequent elimination of transgene is a major limitation of adenovirus gene therapy. ${ }^{1,2}$ These events occur during the first few weeks after Ad administration. ${ }^{3}$ The immune response to Ad consists first of an innate immune response followed by a specific $\mathrm{CD}^{+}$cytotoxic $\mathrm{T}$ lymphocyte (CTL) response and production of neutralizing antibody. ${ }^{4}$ The antigenspecific CTL response is thought to have a major role in the elimination of cells transfected with the Ad vectors that express the transgene. ${ }^{5,6}$ However, the relative contribution of the specific CTL response and innate immune responses to the clearance of Ad and associated transgenes has been a subject of debate.

There is clear evidence that for some viruses such as vaccinia virus and lymphocytic choriomeningitis virus (LCMV), the CD8 ${ }^{+}$CTL response is necessary for viral clearance following primary infection. ${ }^{7,8}$ However, the evidence for CTL involvement for clearance of adeno-

Correspondence: Dr JD Mountz, Department of Medicine, Division of Clinical Immunology and Rheumatology, University of Alabama at Birmingham, 701 S. 19th Street, Room 473 LHRB, Birmingham, AL 35294-0007, USA

Received 10 June 2004; accepted 22 December 2004; published online 7 April 2005 virus during a primary response is less certain. In fact, several previous studies showed that the adenovirus genome is rapidly cleared within 4-6 days after inoculation, well before the generation of potent CTLs. ${ }^{9-11}$ The elevation of liver enzymes occurs as a result of the acute response and peaks by day 4 after Ad administration. ${ }^{12}$ Also, we have previously shown that administration of soluble TNF receptor greatly inhibits transgene clearance and the elevation of liver enzymes. ${ }^{13}$ Thus, unlike the critical role of the CTL response after challenge with a highly replicative virus, the role of the CTL response after Ad gene therapy is not clear.

To detect the numbers and functions of Ad-specific CTLs that might contribute to the clearance of Ad and associated liver enzyme elevation, we developed an Ad5specific tetramer that contains the epitope peptide, E1Bp (192 VNIRNCCYI), ${ }^{14-16}$ in the context of class I MHC, D . We then quantified the primary $\mathrm{CD}^{+} \mathrm{T}$-cell response during the 2-week time period following Ad administration. No Ad-specific $\mathrm{CD}^{+} \mathrm{T}$ cells were detectable before day 5, low levels were present at day 6, and peak levels were observed at day 8 after infection. In contrast, liver viral load and serum alanine transaminase (ALT) levels peaked at day 2 and 4, respectively, and returned to baseline values at day 6 . There was no further clearance of Ad virus or liver cytotoxicity during or 
after the peak of the CTL response. These results indicate that the Ad-specific CTLs do not contribute significantly to the clearance of Ad or liver enzyme elevation after a primary administration of Ad.

\section{Results}

\section{Kinetics of the viral clearance and liver enzyme elevation after Ad infection}

C57BL/6 (B6) mice were injected intravenously (i.v.) with $8 \times 10^{8}$ infectious units (i.u.) of wild-type Ad5. At different times after Ad administration, the level of replicating virus and viral genomes was determined and liver enzyme levels were monitored. There were high levels of Ad viral genome and E1B mRNA copies detectable by day 1 after Ad5 administration with a smaller further increase by day 2 followed by a rapid decrease by days 4 and 6 (Figure 1a). However, the levels of viral DNA and mRNA in the liver did not decrease to pretreatment levels and remained at values that were at least 10-50-fold higher than preadministration values, and were nearly constant from day 8 to day 15 . There was a significant increase in the levels of serum ALT at day 2 , which peaked at day 4 , followed by a significant decline by day 6 after virus administration (Figure 1b). These results indicate that, after Ad administration, the immunologic mechanism for clearance of Ad was initiated by day 2 after administration, peaked on day 4 and was largely downregulated by day 6 .
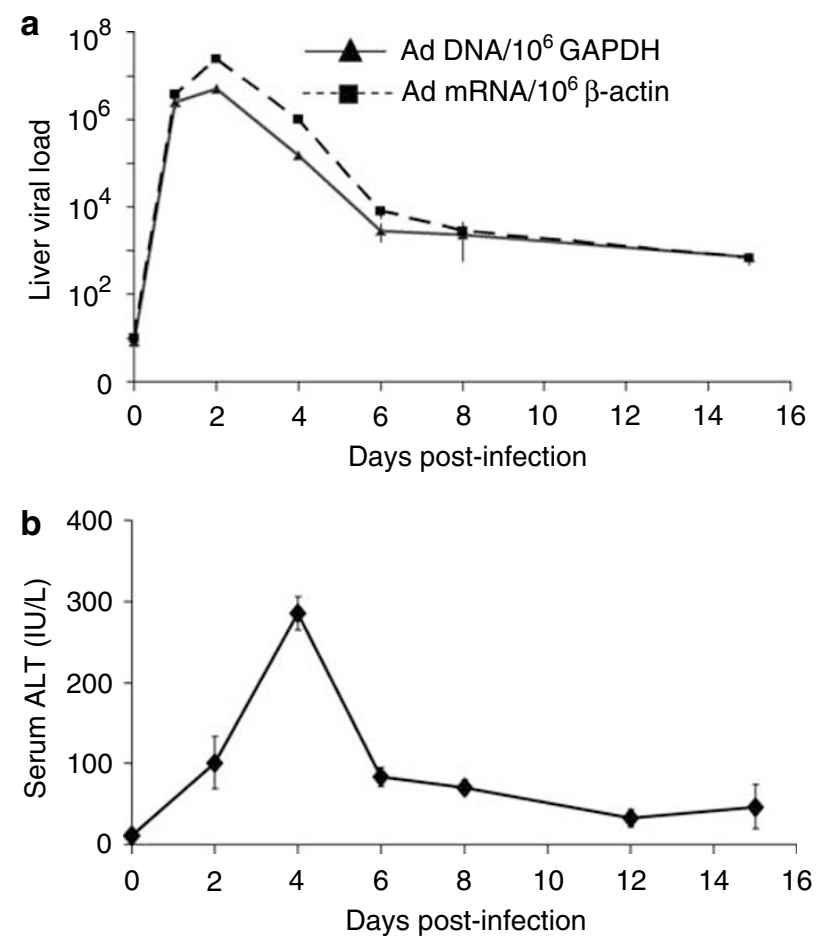

Figure 1 Kinetics of adenoviral clearance from liver and serum ALT elevation. C57BL/6 mice were injected with $8 \times 10^{8}$ i.u. of wild-type Ad5. Livers of B6 mice were recovered at different times after Ad5 infection and processed for total DNA and RNA. Real-time qPCR was performed to

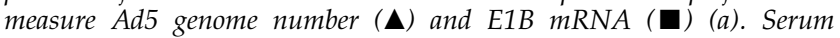
samples were collected at different time points and serum ALT levels were assayed $(b)$. Data shown are the mean \pm s.e.m. of a minimum of three mice at each time point.
Characterization of the primary CD8 $T$-cell response in Ad-primed B6 mice with a virus-specific peptide and a class I MHC tetramer

To determine the role of the virus-specific CD8 T-cell response in the clearance of adenovirus from the liver, we developed an Ad-specific tetramer that enabled detection of Ad-specific CD8 T cells after adenovirus immunization. The immunodominant $\mathrm{H} 2-\mathrm{D}^{\mathrm{b}}$ restricted CTL epitope, E1Bp (192 VNIRNCCYI) ${ }^{14-16}$ is contained in the protein encoded by the Ad E1B gene. The ability of this peptide to stimulate Ad-specific CD8 T cells in the context of class $\mathrm{I} \mathrm{D}^{\mathrm{b}}$ was first demonstrated using an IFN $\gamma$ ELISPOT assay. The E1Bp peptide could specifically stimulate IFN $\gamma$ production by spleen and liver $\mathrm{T}$ cells from B6 mice after injection of Ad5 (Figure 2a). Interestingly, there was a two-fold increase in the number of E1B-specific spot-forming cells (SFCs) from the liver of Ad-infected B6 mice compared to the spleen of Ad-infected B6 mice. As a control, there were very few IFN $\gamma$ spots produced by stimulation of spleen cells from Ad-primed B6 mice by an M1 flu peptide (58 GILGFVFTL), and no production of IFN $\gamma$ spot by spleen or liver cells from naïve B6 mice after stimulation with the E1Bp (Figure 2a).

To determine if the E1Bp peptide could sensitize target cells for lysis by Ad-specific CTLs, spleen cells from naïve B6 mice were either pulsed with the E1Bp peptide and labeled with a high dose of 5,6-carboxysuccinimidylfluoresceine ester (CFSE; CFSE ${ }^{\text {hi }}$ ) or unpulsed and labeled with a low dose of CFSE $\left(\mathrm{CFSE}^{\mathrm{lo}}\right)$. The spleen cells $\left(10^{7} /\right.$ mouse) were then injected i.v. into B6 mice that had been infected with Ad 8 days earlier. The spleens of the recipient mice were recovered $6 \mathrm{~h}$ after injection, a single-cell suspension was prepared and the relative recoveries of CFSE ${ }^{\text {lo }}$ (unpulsed) cells and CFSE ${ }^{\text {hi }}$ (peptide-pulsed) cells were compared. In comparison with unpulsed CFSE ${ }^{\text {lo }}$ cells, 98\% of the E1Bp-sensitized CFSE ${ }^{\text {hi }}$ target cells were deleted in vivo during this 6-h period (Figure $2 b$ ). The lysis was specific for the E1Bp peptide since identical target cells pulsed with the M1 influenza peptide were not eliminated. Similarly, $\mathrm{H} 2-\mathrm{D}^{\mathrm{b}}$-specific lysis was confirmed by the observation that there was no specific lysis of E1Bp pulsed syngenic target cells recovered from $\mathrm{D} 2\left(\mathrm{H}-\mathrm{2}^{\mathrm{d}}\right)$ mice.

Using this Ad-specific peptide, a $\mathrm{D}^{\mathrm{b}}$-E1Bp MHC tetramer was then produced and used to characterize Ad-specific $\mathrm{T}$ cells in the spleen of Ad-injected mice. At 8 days post-infection, $1.7 \%$ of spleen mononuclear cells (MNCs) in the Ad-primed C57BL/6 mice were $\mathrm{D}^{\mathrm{b}}$ $\mathrm{E} \mathrm{Bp}^{+} \mathrm{CD}^{+}$(Figure $2 \mathrm{c}$ ). In contrast, less than $0.01 \%$ of the $\mathrm{CD} 8{ }^{+} \mathrm{T}$ cells in the spleen of naive $\mathrm{B} 6$ mice, or Ad-primed $\mathrm{D} 2$ mice, were $\mathrm{D}^{\mathrm{b}}$-E1Bp tetramer positive. These results show that the E1Bp can be used to detect Ad5-specific CTLs by an ELISPOT assay, by an in vivo killing assay and by tetramer staining.

\section{Ad-specific CD8 T cells in Ad target tissue}

Quantification of Ad-specific CD8 T cells in the blood and target tissues, including the liver and lung, of treated animals would provide valuable information regarding the tissue distribution of CTLs. Through the use of the $\mathrm{D}^{\mathrm{b}}$-E1Bp tetramer, we were able to evaluate the number of Ad-specific MNCs in the peripheral blood (PBMCs) and from the liver and lung. Prior to treatment, less than 
a

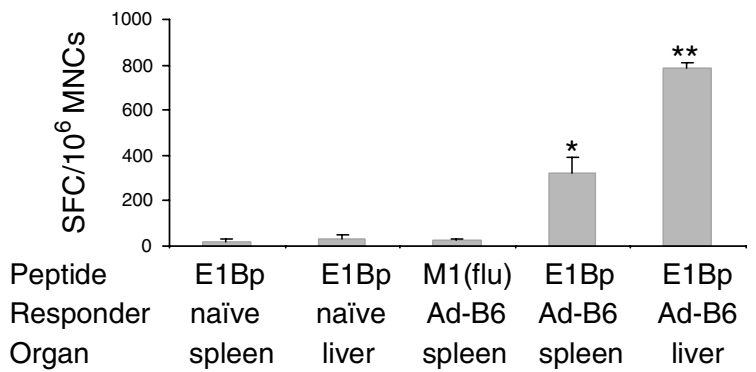

C

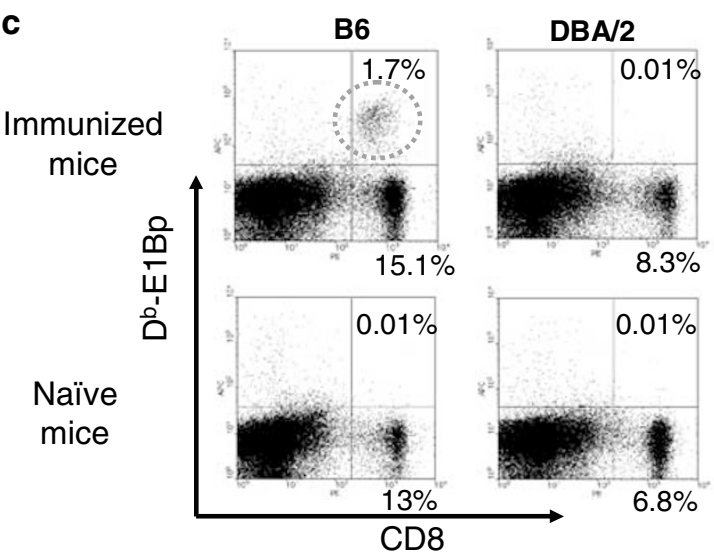

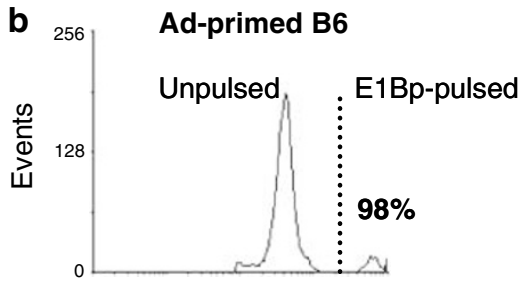

Ad-primed B6

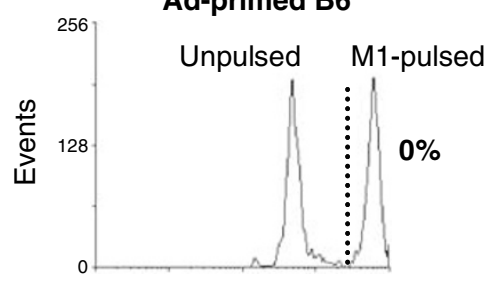

Ad-primed D2

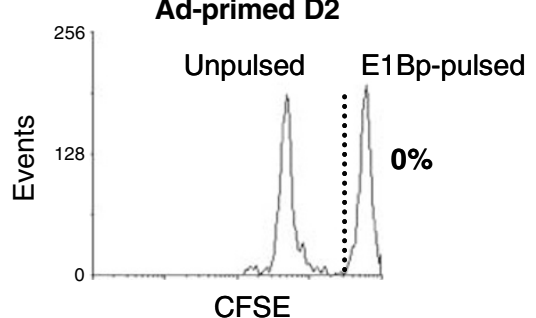

Figure 2 Characterization of epitope peptide E1Bp and MHC tetramer. B6 mice and D2 mice were i.v. injected with $8 \times 10^{8}$ i.u. of wild-type Ad5 and analyzed 8 days later. MNCs from spleens and livers of B6 mice or D2 mice were isolated and IFN $\gamma$ ELISPOT assay was performed as described in Materials and methods. The number of SFC per $10^{6}$ cells was shown (a). Target cells from the spleen of naïve B6 mice were labeled with a high concentration of CFSE $\left(C F S E^{h i}\right)$ and then pulsed either with E1Bp peptide ( $b$; upper panel) or irrelevant flu peptide M1 (b; middle panel). Peptide-pulsed CFSE ${ }^{h i}$ cells were cotransferred i.v. into Ad-primed B6 mice with unpulsed target cells that were labeled with a low concentration of CFSE (CFSE $\left.{ }^{l o}\right)$. Target cells from the spleen of naïve D2 mice were treated similarly and transferred into Ad-primed D2 mice (b; bottom panel). After 6 h, single-cell suspensions of the spleen were analyzed by flow cytometry (100 000 events) for CFSE fluorescence. Numbers in the histograms represent the percentage of target cells killed. MNCs from spleens were labeled with anti-CD8 antibody and $D^{b}$-E1Bp tetramer and analyzed by flow cytometry (c). The results are representative of 5-8 independent experiments. Data shown are the mean \pm s.e.m of SFC $/ 10^{6}$ MNCs from 3-5 mice of each group. ${ }^{*} P<0.05$ and ${ }^{* * P}<0.001$ compared to naïve mice.

$0.01 \%$ of MNCs from the blood, liver and lung of B6 mice were $\mathrm{CD}^{+}$and reactive with the $\mathrm{D}^{\mathrm{b}}$-E1Bp tetramer (Figure 3a, lower panels). At 8 days after treatment, approximately $10 \%(2.0 \% / 20.3 \%)$ of the CD8 ${ }^{+} \mathrm{T}$ cells in the peripheral blood were $\mathrm{D}^{\mathrm{b}}-\mathrm{E} 1 \mathrm{Bp}^{+}$, consistent with the results obtained on analysis of the spleen on day 8 (Figure 3b). Surprisingly, there was extensive recruitment and accumulation of $\mathrm{D}^{\mathrm{b}}-\mathrm{E} 1 \mathrm{Bp}^{+} \mathrm{CD} 8^{+} \mathrm{T}$ cells in the liver and lung. These cells represented approximately $15 \%$ of the total MNCs in the liver and $8 \%$ of the total MNCs in the lung (Figure 3a, upper panels). We found that $33 \%$ of the total $\mathrm{CD}^{+} \mathrm{T}$ cells that accumulate in the liver, and $45 \%$ of the total $\mathrm{CD}^{+} \mathrm{T}$ cells that accumulate in the lung, after administration of adenovirus, were $\mathrm{D}^{\mathrm{b}}$-E1Bp-tetramer positive (Figure $3 \mathrm{~b}$ ). These results indicated that there was marked recruitment of virus-specific $\mathrm{CD}^{+} \mathrm{T}$ cell to the target tissues (liver and lung) following Ad administration.

\section{$D^{b}$-E1Bp-tetramer ${ }^{+} C D 8^{+} T$ cells are cytotoxic effector cells}

To determine the phenotype of the $\mathrm{D}^{\mathrm{b}}-\mathrm{E} 1 \mathrm{Bp} \mathrm{CD}^{+}{ }^{+} \mathrm{T}$ cells in the spleen, MNCs were isolated at day 8 after Ad administration, stimulated in vitro with E1Bp peptide for 3 days, and then were intracellularly co-stained with
anti-IFN $\gamma$ and anti-granzyme B antibodies, respectively. Nearly $100 \%$ of the $\mathrm{D}^{\mathrm{b}}$-E1Bp-specific CD8 T cells were IFN $\gamma^{+}$and granzyme $\mathrm{B}^{+}$(Figure $4 \mathrm{a}$ and $\mathrm{b}$ ). At the same time, all of these $\mathrm{D}^{\mathrm{b}}$-E1Bp-specific CD8 T cells expressed elevated levels of CD44, an activation/memory marker for T cells (Figure 4c), and low levels of CD127 (Figure $4 \mathrm{~d})$, the IL-7 receptor that is essential for T-cell survival and homeostatic maintenance of memory $\mathrm{T}$ cells. ${ }^{17} \mathrm{On}$ day 8 , only $22 \%$ of $\mathrm{D}^{\mathrm{b}}$-E1Bp-specific CD8 $\mathrm{T}$ cells expressed high levels of CD127, indicating that these cells were acute effector cells. After the acute CTL response, the $\mathrm{CD} 127^{\mathrm{hi}}$ population gradually increased to $46 \%$ at day 15 and $63 \%$ at day 200 after infection (Figure $4 d$ ). Therefore, Ad administration is associated with the development of specific effector CD8 T cells and the subsequent generation of Ad-specific memory cells.

\section{Ad-specific CTL response occurs after liver toxicity and virus clearance}

To address whether the Ad-specific CTL response correlates with the clearance of Ad or liver enzyme elevation, we utilized $\mathrm{D}^{\mathrm{b}}-\mathrm{E} 1 \mathrm{Bp}$ tetramers and E1Bpspecific in vivo CTL assay. MNCs from the spleen, liver and lung were obtained on days $0,3,6,7,8,10,15$, and 30 after i.v. administration of Ad5. As shown in Figure 5a, 

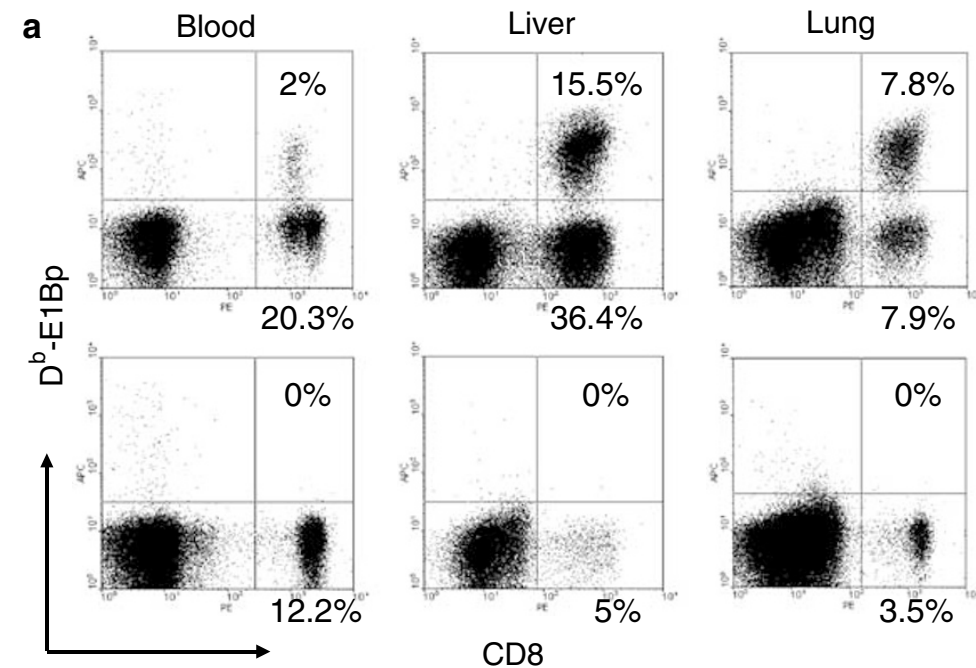

Immunized
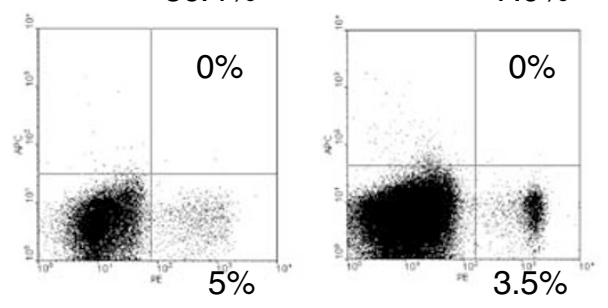

Naive

b

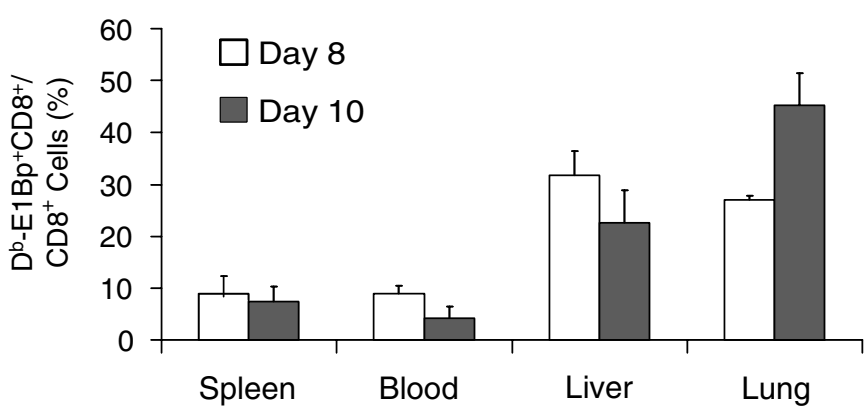

Figure $3 D^{b}$-E1Bp tetramer staining of MNCs from blood, liver and lung. MNCs from peripheral blood, liver and lung were isolated from B6 mice at day 8 post-infection. All cells were labeled with $D^{b}$-E1Bp tetramer and anti-CD8 antibodies and analyzed by flow cytometry (a). The percentage of $D^{b}$-E1B $p^{+}$cells in total $C D 8^{+} T$ cell population of the spleen, blood, liver and lung at days 8 and 10 post infection are shown in (b). Data shown are the mean \pm s.e.m. of $D^{b}-E 1 B p^{+} C D 8^{+}$cells/total CD8 ${ }^{+} T$ cells from 3-5 mice each group.

there were no detectable tetramer-positive cells in the spleen, liver and lung at day 3 and a very low percentage at day 6, a time when the clearance of Ad had been substantially accomplished and after the peak of liver enzyme elevation. The tetramer-positive cells began to increase after day 6, sharply increased and peaked at day 8 and declined thereafter (Figure 5a). This did not correlate with a further reduction of Ad-viral clearance and liver enzyme elevation shown in Figure 1. Therefore, the primary peak response of tetramer ${ }^{+} \mathrm{CD} 8 \mathrm{~T}$ cells does not correlate with the elimination of Ad or the liver cytotoxic response.

To determine if the cytotoxic activity in the spleen and liver correlated with the presence of the tetramerpositive CD8 T cells, the kinetics of the specific in vivo CTL activity against CFSE-labeled, E1Bp-pulsed target cells was measured. The E1Bp-specific CTL activity in spleen and liver was low at day 3 (Figure 5b). The CTL activity was detectable at day 6 , peaked at day 8 and declined by day 10 . These results indicate that the peak of the Ad-specific CTL response at day 8 occurred after clearance of $\mathrm{Ad}$ and after the peak of liver cell damage.

\section{Kinetics of Ad viral clearance and liver enzyme elevation in SCID mice}

The above results indicate that, after a primary challenge, the Ad viral clearance and liver cell toxicity occur before the peak of the primary $\mathrm{CD} 8^{+} \mathrm{CTL}$ response. This was further confirmed by administration of wild-type Ad5 to B6.SCID (H-2 $\left.{ }^{\mathrm{b}}\right)$ mice and subsequent analysis of Ad-viral clearance and serum ALT levels at different times. The kinetics of Ad clearance and serum ALT elevation were identical in SCID mice and wild-type B6 mice. For both strains, Ad-virus genome and serum ALT peaked at day 2 and day 4, respectively, and declined thereafter (Figure $6 a$ and $b)$. As expected, there were no tetramer-positive $T$ cells generated after administration of Ad5 into SCID mice (Figure 6c). There was also no in vivo CTL response to E1Bp-pulsed target cells after being transferred into SCID 8 days after Ad5 administration (data not shown). The results above indicate that this early immune response might completely account for the clearance of Ad and liver toxicity.

Serum levels of IFN $\gamma$ were analyzed at different times after Ad5 administration. The serum levels of IFN $\gamma$ peaked at day 2 after Ad administration and declined gradually thereafter (Figure 6d). These results suggest that innate immune response, but not the virus-specific CD8 T cells, account for Ad clearance and liver cell damage.

\section{Discussion}

The CTL response to Ad vectors has been proposed to play a key role in the elimination of transduced cells, thus limiting transgene expression. ${ }^{5,18}$ Immunosuppres- 
a

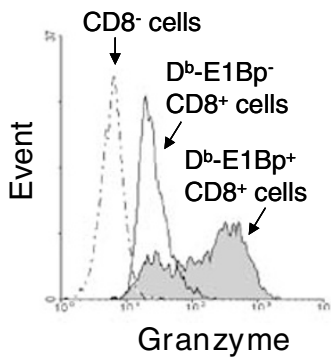

C

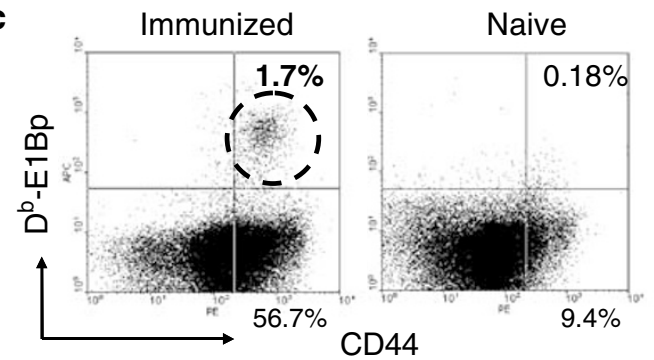

b

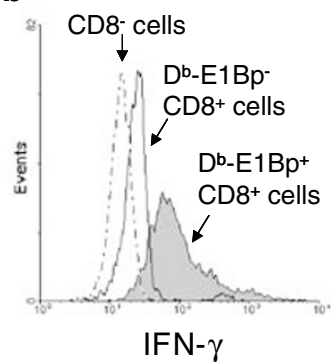

CD44

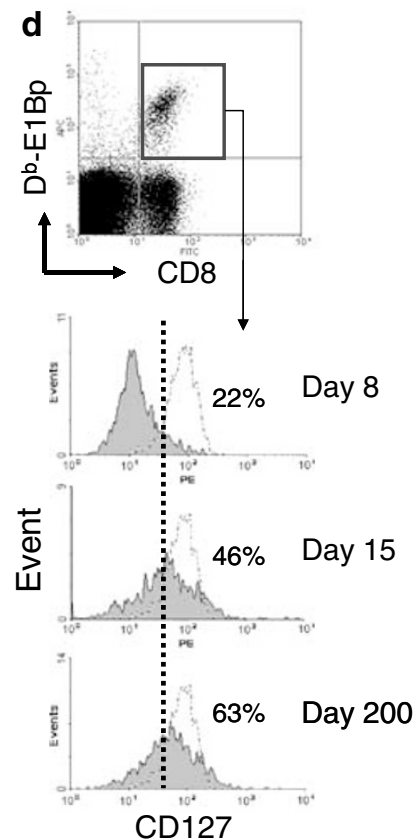

Figure 4 Characterization of $D^{b}-E 1 B p^{+} C D 8^{+}$cells. $B 6$ mice were i.v. injected with wild-type Ad5 and killed 8 days later. Spleen cells from B6 mice at day 8 were incubated in vitro with E1Bp-pulsed, irradiated spleen cells from naïve B6 mice for 3 days and then intracellularly labeled with anti-granzyme B (a) or anti-IFN $\gamma(b)$ antibodies. Spleen cells were stained with anti-CD44 along with anti-CD8 antibodies and $D^{b}$-E1Bp tetramer 8 days post-infection. Spleen cell labeled with CD44 and tetramer were gated on $C D 8^{+}$cells (c). The numbers in the right upper quadrants indicate the percentage of CD8 T cells that were tetramer ${ }^{+}$. CD127 (IL-7R $\left.\alpha\right)$ expression on spleen cells recovered from B6 mice at days 8,15 and 200 were analyzed (d). Histograms were gated on $D^{b}-E 1 B p^{+}$ $C D 8^{+}$cells. The numbers in the histograms indicate the percentage of $C D 127^{\text {hi }}$ cells. The shaded area indicates the $D^{b}$-E1Bp ${ }^{+} C D 8^{+}$cells and dotted lines indicate the $\mathrm{Db}-\mathrm{E} 1 \mathrm{Bp}{ }^{-} \mathrm{CD} 8^{+}$cells recovered from naïve mice.

sive strategies have been proposed to prolong therapeutic gene expression and prolong gene therapy efficacy, which have been reviewed elsewhere. ${ }^{1,2}$ However, until now, the analysis of the CTL response could only be explored using in vitro assays or through indirect approaches. ${ }^{19-22}$ Consequently, the mechanisms underlying CTL generation and effector functions after $\mathrm{Ad}$ infection have not been clarified completely, making it difficult to correlate specific CTL generation with Ad virus clearance and liver toxicity. The primary limitation of the in vitro CTL assay is that in vitro killing activity can be induced by several specific effector mechanisms including the cytolytic molecules TNF $\alpha$ and IFN $\gamma$, in addition to the perforin/granzyme-B lysis induced by effector cells that act on specific and/or bystander targets. Thus, it is not possible to obtain a precise relationship between the numbers of effector cells which can execute specific cytotoxicity based on the results of in vitro assays. Since the MHC class I/peptide tetramer technology was first introduced in $1996,{ }^{23}$ it has been widely applied in the quantification and characterization of $\mathrm{CD}^{+} \mathrm{T}$ cells induced by viral and bacterial infections. ${ }^{24}$ We have now used this tetramer-binding method to gain a better understanding of the role of CTL response after Ad administration. We also applied an in vivo CTL assay to directly investigate and distinguish the generation and effector functions of Ad-specific CD8 T cells.

The Ad E1B gene-encoded protein contains the immunodominant CTL epitope peptide (192 VNIRNCCYI), referred to as E1Bp, that is presented by the $\mathrm{H}_{2}-\mathrm{D}^{\mathrm{b}}$ molecule. ${ }^{14-16}$ We developed the tetramer, $D^{\mathrm{b}}-\mathrm{E} 1 \mathrm{Bp}$, consisting of $\mathrm{H} 2-\mathrm{D}^{\mathrm{b}}, \mathrm{E} 1 \mathrm{Bp}$, and $\beta_{2}$-microglobulin that is conjugated with the flurochrome, allophycocyanin (APC). Analysis of the specificity of this $\mathrm{D}^{\mathrm{b}}-\mathrm{E} 1 \mathrm{Bp}$ tetramer in Ad-immunized B6 mice $\left(\mathrm{H}-2^{\mathrm{b}}\right)$, naïve mice, and Ad-immunized D2 mice $\left(\mathrm{H}-2^{\mathrm{d}}\right)$ indicated that 8 days after immunization, approximately $10 \%$ of $\mathrm{CD}^{+} \mathrm{T}$ cells in the spleen were specific for E1Bp epitope in B6 mice. The tetramer-positive $\mathrm{CD} 8^{+} \mathrm{T}$ cells expressed elevated levels of CD44, an activation/memory marker of lymphocytes, and were nearly $100 \% \mathrm{IFN} \gamma^{+}$and granzyme- $\mathrm{B}^{+}$. The numbers of effector cells exhibited similar kinetics in the liver. It has been proposed that IL-7 is essential for both naïve and memory T-cell survival, ${ }^{25,26}$ and IL-7 receptor deprivation in effector CTLs ensures their death after viral clearance. ${ }^{27}$ IL-7R $\alpha$-chain (CD127) has been used as a marker to distinguish primary effector cells from memory T-cell precursors. ${ }^{17}$ In the present study, $78 \%$ of $\mathrm{D}^{\mathrm{b}}-\mathrm{E}^{\mathrm{BBp}} \mathrm{P}^{+} \mathrm{CD} 8 \mathrm{~T}$ cells expressed low levels of CD127 during the peak of the CTL response, indicating these cells would not develop into memory effector CTLs, but rather undergo apoptosis. ${ }^{28}$ The peak of the virus clearance and liver enzyme elevation occurred by day 4 , before a significant number of Adspecific CTLs were generated, indicating that the innate immune response is responsible for most of the virus clearance after a primary response. These results are consistent with previous findings that there was an identical decrease in adenoviral DNA in the liver and lung of both immunocompetent and immunodeficient mice. ${ }^{9,29,30}$ In those cases, Ads were replication-defective due to deletion of the $E 1$ and/or $E 4$ genes, and it was not surprising that more than $80 \%$ of viral DNA was eliminated in the first hours following infection. In the present study, replicative wild-type Ad was used and the 

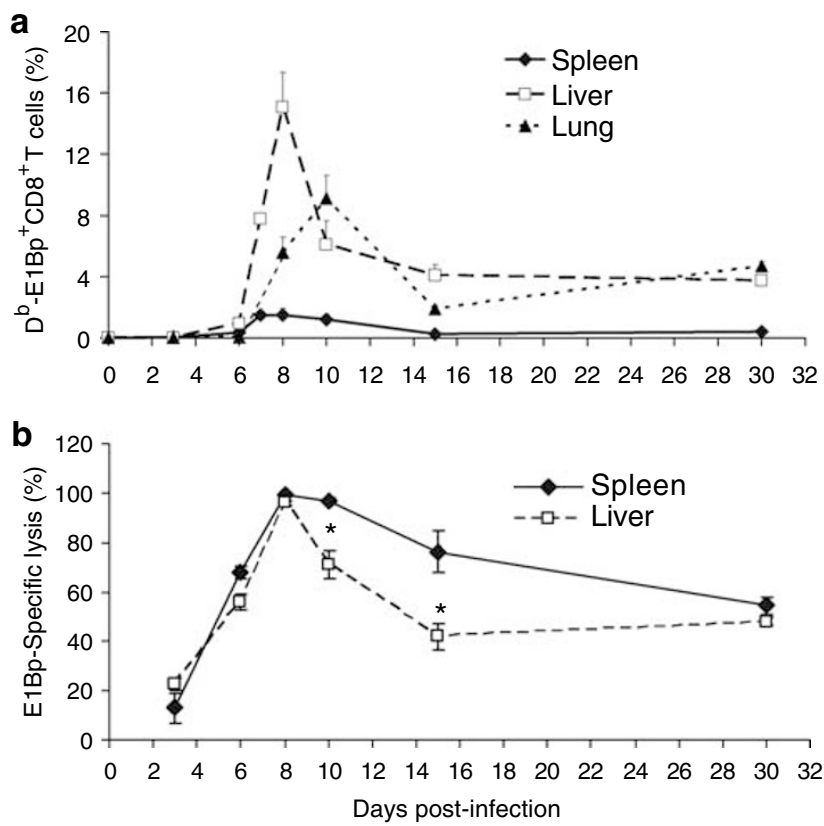

Figure 5 Kinetics of Ad-specific CTL response. B6 mice were i.v. injected with wild-type Ad5. The Ad-specific CTL response was analyzed by $D^{{ }^{-}}$E1Bp tetramer and in vivo CTL assay at indicated days post-infection. The frequencies of $D^{b}-E 1 B p^{+} C D 8^{+}$cells in the spleen, liver and lung are indicated for each time point (a). Target cells from the spleen of naive B6 mice were labeled with a high concentration of CFSE (CFSE hi ) and then pulsed with E1Bp peptide. Peptide-pulsed CFSE ${ }^{\text {hi }}$ cells were cotransferred i.v. into adenovirus-primed B6 mice with unpulsed target cells that were labeled with a low concentration of CFSE (CFSE $\left.{ }^{l o}\right)$. After $6 \mathrm{~h}$, single-cell suspensions of the spleen and liver were analyzed by flow cytometry (100000 events) for CFSE fluorescence. E1Bp-specific lysis at the indicated time points was calculated as described in Materials and methods and is shown in (b). Data shown are the mean \pm s.e.m. of 3-5 mice of each group. ${ }^{*} \mathrm{P}<0.05$ for lysis in livers compared to spleens.

viral DNA and E1B mRNA in the liver were greater on day 2 than day 1 , indicating moderate replication of the virus in vivo.

There were detectable, stable levels of virus and virus DNA in the liver during and after the peak of the CTL response. This is consistent with a previous report showing that Ad vector infected cells can escape CTL lysis in vivo. ${ }^{31}$ It was also noted that after influenza virus infection, the number of apoptotic $\mathrm{CD}^{+} \mathrm{T}$ cells was higher in the liver, suggesting that antigen-specific CD8 ${ }^{+}$ $\mathrm{T}$ cells were destroyed in the liver. ${ }^{32}$ Downregulation of antigen presentation in the liver would make these liver cells resistant to CTL response. ${ }^{33}$ We propose that viral persistence in the liver during and after the peak CTL response is due to the inability of infected liver cells to form good targets for CTL attack. We also observed that the killing efficiency of CTLs in the liver for peptidepulsed target cells was lower compared to the spleen. This suggests that other mechanisms were involved in limiting the duration of antigen-specific cytotoxicity in the liver. This was supported by our previous findings that soluble Fas could inhibit the CTL response in the liver. ${ }^{34} \mathrm{CD} 4{ }^{+} \mathrm{CD} 25^{+}$regulatory $\mathrm{T}$ cells have also been proposed to play an inhibitory role in suppression of CTLs in the liver. ${ }^{35}$

The clearance of the Ad and associated transgene from the liver has been proposed to be due to the effects of cytokines, such as TNF $\alpha$ and IFN $\gamma$, which may be associated with both the CTL response and the innate immune response. ${ }^{36}$ In the present study, the peak of the CTL response occurs after viral clearance and liver enzyme elevation indicating viral clearance and enzyme elevation are primarily due to the innate immune response. We therefore propose that the development of the CTL response was set in motion by early immune response mechanisms, but the presence of detectable antigen was not necessary when the CTL response reached its peak. ${ }^{37}$ In perforin knockout mice, we and others have shown that there was a poor viral clearance in the absence of perforin, ${ }^{38,39}$ indicating a key role for perforin release from CTLs to mediate viral clearance. The lack of a significant role for the CTL response in the clearance of the Ad5 in the present study may be due to the relatively low replication of the virus. The virus reached a high titer on day 1 , peaked on day 2 , then declined to a maintenance level by day 6 . The kinetics of viral clearance is consistent with previous work with the E1-deleted, first-generation Ad vector or helper-dependent Ad vector. ${ }^{11}$ It was also shown that after exposure to hepatitis $\mathrm{C}$ by a cutaneous route, the CTL response was not required to eliminate hepatitis $C$, whereas, after i.v. acquisition, the CTLs were required..$^{40}$ Together, the results suggest that under conditions of low virus load or low viral replication, as in these experiments, the CTL response may not play a critical role in clearance of the virus during the primary immune response.

One of the major obstacles facing gene therapy is not only to permit long-term expression of the transgene by minimizing the primary immune responses, but also to predict the pre-existing immune response of the individual to gene therapy. A recent survey including Europe, Japan, and the United States showed a $>80 \%$ pre-exposure to the most commonly used Ad5 virus ${ }^{41}$ in the adult population (age range from 20 to 70 years old). This will present a problem for Ad5-based gene therapy in these individuals. Therefore, one important application of tetramer technology would be to predict the magnitude of the CTL response that might occur upon administration. As an example, the possible epitope peptides from Ad5 hexon protein were predicted to be 913-921 TLLYVLFEV for HLA A0201* with two prediction programs. $^{42,43}$ Its antigenicity had been shown to specifically stimulate IFN $\gamma$ production of CD8 $\mathrm{T}$ cells from HLA-A0201* donors. ${ }^{44}$ In combination with everincreasing knowledge of T-cell epitopes of $\mathrm{Ad}$, the present results demonstrate the feasibility of development of class I MHC/peptide tetramers in humans with different MHCs.

The second potentially clinical application of the present work is the demonstration that the use of a tetramer combined with the analysis of CD127 (IL-7 receptor $\alpha$-chain) expression can predict the magnitude of the CTL response. Following acute exposure of naïve mice, there is homeostatic maintenance of memory CD8 $\mathrm{T}$ cells that are derived from the CD127 ${ }^{\mathrm{hi}}$ population. ${ }^{17}$ This population is predicated to give rise to the highest number of CTLs upon re-challenge. Therefore, the combined analysis of PBMCs from humans with an Ad-specific tetramers and the IL-7R antibody, antiCD127 will provide important predictive information as to the magnitude of the CTL response. We would predict that patients with the highest number of 
a

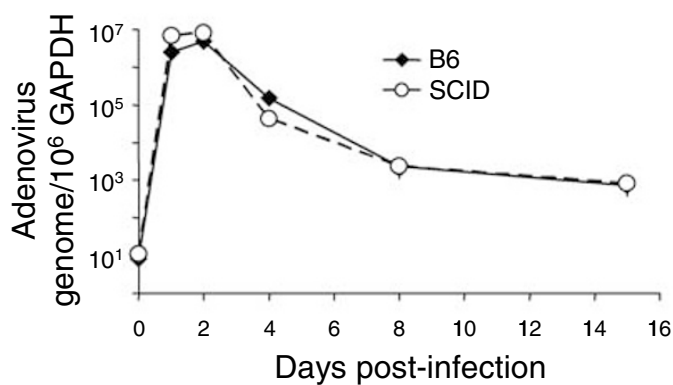

C

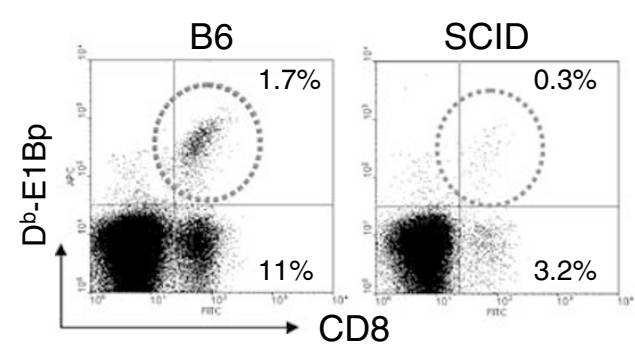

b
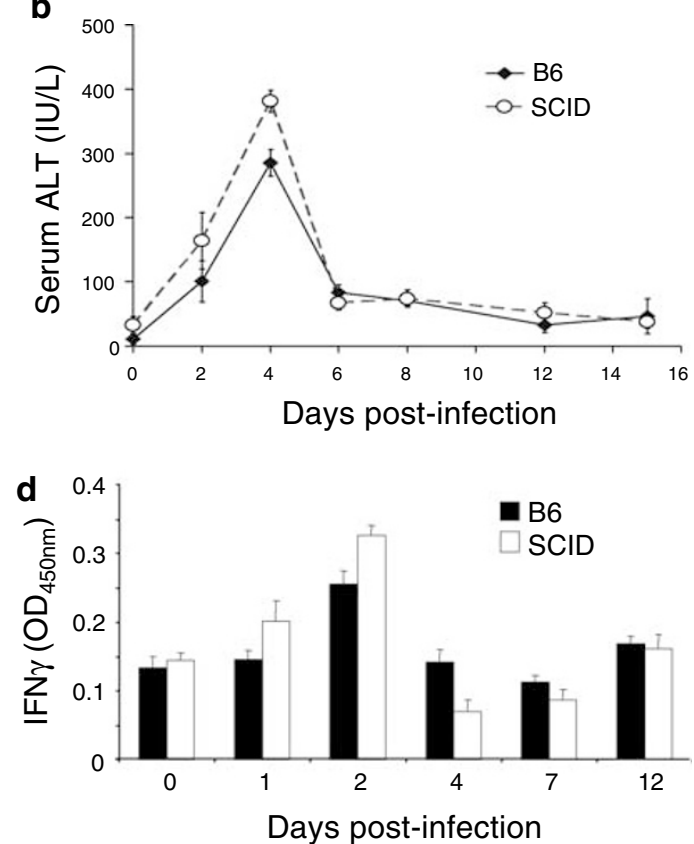

Figure 6 Analysis of the immune response to Ad infection in SCID mice. B6; SCID $\left(H-2^{b}\right)$ and wild-type B6 mice were i.v. injected with $8 \times 10^{8}$ i.u. of wild-type Ad5 and analyzed at indicated days post-infection. Livers of SCID and B6 mice were recovered at different times after Ad5 infection and processed for total DNA isolation. Real-time qPCR was performed to measure Ad5 genome number (a). Serum samples of SCID and B6 mice were collected at indicated days and serum ALT was assayed (b). Spleen MNCS of SCID and B6 mice were labeled with anti-CD8 Ab and Db-E1Bp tetramer 8 days postinfection and analyzed by flow cytometry (c). Serum IFN $\gamma$ levels in SCID and B6 mice at each time point post-infection were measured by ELISA (d). The results are representative of 5-8 independent experiments. Data shown are the mean \pm s.e.m. of 3-5 mice at each time point.

tetramer-positive and CD127 ${ }^{\text {hi }} \mathrm{T}$ cells would be at especially high risk for forming a brisk antivirus CTL response upon re-challenge. Armed with this knowledge, gene therapy could be individually tailored to patients with different HLA haplotypes to avoid a potential memory $\mathrm{T}$-cell response.

\section{Materials and methods}

\section{Mice}

Female C57BL/6 (B6; $\mathrm{H}-2^{\mathrm{b}}$ ), B6.SCID (SCID; $\mathrm{H}-2^{\mathrm{b}}$ ), and DBA/2 (D2; H-2 $\left.{ }^{\mathrm{d}}\right)$ mice at $6-8$ weeks of age were obtained from The Jackson Laboratory (Bar Harbor, ME, USA). Groups of at least 3-5 mice were analyzed in each experiment or at each time point. All animal protocols were approved by the Institutional Animal Care and Use Committee at University of Alabama at Birmingham.

\section{Ad and infection}

Wild-type Ad type 5 was obtained from the American Tissue Culture Collection (Rockville, MD, USA) and propagated in the HEK293 cells as described previously. ${ }^{13}$ Infected cells were harvested and lysed by three freeze-thaw cycles to release the virus, which was then purified through two cesium chloride gradients. The purified wild-type Ad5 was then titrated by the plaque assay, ${ }^{45}$ aliquoted, and stored at $-80^{\circ} \mathrm{C}$ until used. Mice were administered at $8 \times 10^{8}$ i.u. per mouse through tail vein injection on day 0 and killed at different time points for CTL analysis.

\section{Cell preparation}

Single-cell suspensions of spleen and lung were prepared as described previously. ${ }^{13}$ Briefly, spleens and lungs were disrupted using wire mesh screens, and erythrocytes were lysed by treatment with buffered$\mathrm{NH}_{4} \mathrm{Cl}\left(8.29 \mathrm{~g}\right.$ of $\mathrm{NH}_{4} \mathrm{Cl}, 1.0 \mathrm{~g}$ of $\mathrm{KHCO}_{3}$, and $0.037 \mathrm{~g}$ of EDTA/1). The livers were strained through a $70 \mu \mathrm{m}$ nylon cell screen (BD Falcon, Bedford, MA, USA). Cells were collected and MNCs were centrifuged at 1500 r.p.m. for $5 \mathrm{~min}$. The cell pellets were resuspended in $8 \mathrm{ml} 40 \%$ Percoll, pipetted onto $3 \mathrm{ml} \mathrm{70 \%} \mathrm{Percoll} \mathrm{and} \mathrm{centrifuged}$ at 2500 r.p.m. for $20 \mathrm{~min}$ at room temperature. The cells were washed by PBS containing 2\% (w/v) BSA and $0.2 \%$ $(\mathrm{w} / \mathrm{v}) \mathrm{NaN}_{3}$ before staining with fluorescent antibodies.

\section{Determination of serum ALT}

Blood samples from injected and control mice, drawn from the retro-orbital sinus at the indicated time points, were centrifuged for serum isolation. Serum samples were analyzed for alanine aminotransferase (ALT) level, a marker of hepatocellular damage, using a colorimetric ALT detection kit (TECO Diagnostics, Anaheim, CA, USA) according to the manufacturer's protocol without modifications. ALT levels were measured in triplicate by using plasma samples obtained from at least three mice.

\section{$R T-P C R$}

Total RNA was isolated from the liver of infected mice at different time points by a single step method using TRIzol reagent (Life Technologies, Gaithersburg, MD, USA) according to the manufacturer's instructions. Thereafter, RNA was transcribed to cDNA with a first- 
strand cDNA synthesis kit (Amersham Pharmacia Biotech, Piscataway, NJ, USA).

\section{Real-time PCR}

For detection of Ad type 5, the following forward and reverse primers specific for hexon gene, respectively,

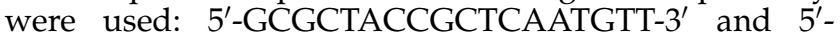
AGGCACCTGGATGTGGAA- $3^{\prime}$. The mouse GAPDH gene was detected as internal control with the forward primer, 5'-AGCCGCATCTTCTTGTGC-3', and reverse primer, 5'-TCCGTTCACACCGACCTT-3'. To detect E1B CDNA, the following primers were used: forward, $5^{\prime}-$ AGACACAAGAATCGCCTGCT- $3^{\prime}$ and reverse, $5^{\prime}$ CTGCTCCTCCGTCGGTATTA-3'. Mouse $\beta$-actin cDNA was used as internal control with the forward primer, 5'-GACGGCCAGGTCATCACTAT-3' and reverse primer, 5'-AAGGAAGGCTGGAAAAGAGC-3'. The reaction mixture contained SYBR Green PCR Master Mix (Applied Biosystems, Foster City, CA, USA) with $100 \mathrm{ng}$ RNase-treated DNA, 25 pmol of each primer, and in $25 \mu \mathrm{l}$ volumes. Reactions were performed in ABI Prism 7000 Sequence Detection System (Applied Biosystems) with thermal cycling conditions as follows: $50^{\circ} \mathrm{C}$ for $2 \mathrm{~min}$, then a $95^{\circ} \mathrm{C}$ hold for $10 \mathrm{~min}$, followed by 40 cycles at $95^{\circ} \mathrm{C}$ for $20 \mathrm{~s}$, and $60^{\circ} \mathrm{C}$ for $1 \mathrm{~min}$. For each sample, DNA copies of the Ad5 hexon gene were normalized for DNA loading using the number of DNA copies of the mouse $G A P D H$ gene and the results were reported as the number of Ad genome copies per $10^{6} \mathrm{GAPDH}$ genome copies. Ad5 E1B cDNA copies were normalized to $\beta$-actin cDNA content and expressed as the number of E1B mRNA copies per $10^{6} \beta$-actin cDNA copies.

\section{IFNY ELISPOT}

To determine the frequency of peptide epitope-specific $\mathrm{T}$ cells in the spleen and liver, IFN $\gamma$ ELISPOT assay was performed. 96-well polyvinylidene difluoride-backed plates (Millipore, Bedford, MA, USA) were coated overnight with $5 \mu \mathrm{g} / \mathrm{ml}$ anti-mouse IFN $\gamma \mathrm{mAb}$ at $4^{\circ} \mathrm{C}$, washed, and then blocked with complete medium. Cells from spleens or livers of naïve or immunized mice were washed and adjusted to a concentration of $2 \times 10^{6} / \mathrm{ml}$ in complete medium, then added to the microtiter wells together with either $10 \mu \mathrm{g} / \mathrm{ml}$ Ad E1Bp or human influenza virus $\mathrm{M} 1$ peptide (58 GILGFVFTL) to give a final volume of $200 \mu \mathrm{l}$ containing $2 \times 10^{5}$ cells; control wells received no peptide. After 3 days incubation, cells were washed three times with PBS/0.05\% Tween 20, before overnight incubation at $4^{\circ} \mathrm{C}$ with $1 \mu \mathrm{g} / \mathrm{ml}$ biotinylated anti-mouse IFN $\gamma \mathrm{mAb}$ in PBS/10\% FCS. Plates were washed three times with PBS/0.05\% Tween 20, before adding $40 \mathrm{ng}$ of HRP-conjugated goat anti-biotin $\mathrm{Ab}$ (Vector Laboratories, Burlingame, CA, USA), in $100 \mu \mathrm{l}$ of PBS $/ 10 \%$ FCS, to each well. Plates were incubated at room temperature for $1 \mathrm{~h}$, and washed with PBS/0.05\% Tween 20 and then with PBS alone before developing spots using 3-amino-9-ethylcarbazole. Plates were read on a CTL automatic ELISPOT reader (CTL, Cleveland, $\mathrm{OH}, \mathrm{USA}$ ) and analyzed using Immunospot 3.1 software (CTL). All results were repeated in triplicate.

\section{Preparation of MHC class I tetramers}

MHC class I tetramers were prepared, as described previously. ${ }^{46}$ Briefly, $\beta_{2}$-microglobulin as well as recombinant $\mathrm{H}-2 \mathrm{D}^{\mathrm{b}} \mathrm{H}$ chains fused to a BirA substrate peptide were produced in Escherichia coli BL21 (DE3). Monomeric MHC-peptide complexes were refolded with the Ad5 peptide epitope E1Bp (192 VNIRNCCYI) and subsequently enzymatically biotinylated using BirA. Tetramers were formed by the stepwise addition of APCconjugated streptavidin (Molecular Probes, Eugene, OR, USA).

\section{MHC class I tetramer staining}

MNCs from spleen, lung, liver and peripheral blood were stained with APC-conjugated MHC class I tetramer, $\mathrm{D}^{\mathrm{b}}$-E1Bp, anti-CD8 FITC and anti-CD44 PE or antiCD127 PE (BD PharMingen, San Diego, CA, USA). Staining procedures were performed at $4{ }^{\circ} \mathrm{C}$ in PBS containing 2\% (w/v) BSA and $0.2 \%(\mathrm{w} / \mathrm{v}) \mathrm{NaN}_{3}$. After incubation with the antibodies, the samples were washed and then fixed in PBS containing 2\% (w/v) paraformaldehyde. At least 100000 events were acquired using a FACSCalibur (BD Biosciences, San Jose, CA, USA) and analyzed using the computer program CellQuest.

\section{Intracellular cytokine staining}

MNCs from spleen or liver were either left untreated or stimulated with E1Bp peptide $(10 \mu \mathrm{g} / \mathrm{ml})$ for 3 days at $37^{\circ} \mathrm{C}$. Before intracellular staining, cells were stained with $\mathrm{D}^{\mathrm{b}}$-E1Bp tetramer and anti-CD8 PE as described above. After being fixed and permeabilized with BD Cytofix/Cytoperm solution (BD Biosciences), cells were stained with anti-granzyme $\mathrm{B}^{28}$ (BD Pharmingen) or anti-IFN $\gamma$ (eBioscience) Abs per the manufacturer's instruction.

\section{In vivo CTL assay}

For target cells, $10^{7}$ spleen cells from naïve B6 mice were incubated with either $2 \mu \mathrm{M}$ (high dose) CFSE (CFSE ${ }^{\mathrm{Hi}}$ ) or $0.2 \mu \mathrm{M}$ (low dose) CFSE (CFSE ${ }^{\mathrm{Lo}}$ ) in PBS, at $25^{\circ} \mathrm{C}$ for 12 min. CFSE labeling was then quenched by the addition of fetal bovine serum (FBS) to a final concentration of $20 \%(\mathrm{v} / \mathrm{v})$. Cells were washed with RPMI-1640 supplemented with $10 \%$ FBS, $100 \mathrm{U} / \mathrm{ml}$ penicillin/ streptomycin, $2 \mathrm{mM}$ L-glutamine, $50 \mu \mathrm{M} \quad \beta$-mercaptoethanol and $25 \mathrm{~mm}$ HEPES. The CFSE ${ }^{\text {hi }}$ cells were then incubated in the presence of $1 \mu \mathrm{M}$ E1Bp peptide at $37^{\circ} \mathrm{C}$ for $1 \mathrm{~h}$, whereas $\mathrm{CFSE}^{\text {lo }}$ cells were incubated in medium only. Human influenza virus M1 peptide (58 GILGFVFTL) was used as control to pulse a separate population of CFSE ${ }^{\text {hi }}$ cells. After labeling and peptide pulsing, both populations of target cells were washed and mixed together in ice-cold PBS such that recipient mice received $10^{7}$ cells of each population in a single i.v. injection. As a control, naïve B6 mice were adoptively transferred with an identical number of target cells. Recipient mice were killed $6 \mathrm{~h}$ following cell transfer, spleen and liver MNCs were prepared as described above and then analyzed by flow cytometry. Percent specific lysis was determined using the following formulae: ${ }^{47}$

The ratio of recovery of nonpeptide-treated control spleen cells to peptide-sensitized spleen cells = (percentage of CFSElo cells)/(percentage of CFSE ${ }^{\text {hi }}$ cells).

The percent specific lysis $(\%)=100 \times(1-($ ratio of cells recovered from naive mice/ratio of cells recovered from infected mice)). 


\section{Serum ELISA}

Before and at days 1, 2, 4, 7 and 12 after Ad5 injection, serum samples were collected from $\sim 50 \mu \mathrm{l}$ of blood obtained from mice via retro-orbital bleeds, and assayed on ELISA plates coated with capture antibodies for IFN $\gamma$. All subsequent steps in the ELISA were performed according to the manufacturer's instructions (eBioscience).

\section{Statistical analyses}

Significant differences between mean values were determined by Student's $t$-test. $P<0.05$ as considered statistically significant.

\section{Acknowledgements}

We thank Dr Fiona Hunter for reviewing the manuscript, Dr Rafi Ahmed for providing critical suggestions, and Carol Humber for excellent secretarial work. This work was supported in part by National Institutes of Health Grants RO1-AI-42900 (to JD Mountz) and RO1-CA-86881 (to DT Curiel), and by a VA Career Development Award and Merit Review Award (to JD Mountz).

\section{References}

1 St George JA. Gene therapy progress and prospects: adenoviral vectors. Gene Therapy 2003; 10: 1135-1141.

2 Jooss $\mathrm{K}$, Chirmule $\mathrm{N}$. Immunity to adenovirus and adenoassociated viral vectors: implications for gene therapy. Gene Therapy 2003; 10: 955-963.

3 Hackett NR, Kaminsky SM, Sondhi D, Crystal RG. Antivector and antitransgene host responses in gene therapy. Curr Opin Mol Ther 2000; 2: 376-382.

4 Bromberg JS, Debruyne LA, Qin L. Interactions between the immune system and gene therapy vectors: bidirectional regulation of response and expression. Adv Immunol 1998; 69: 353-409.

5 Yang Y, Ertl HC, Wilson JM. MHC class I-restricted cytotoxic T lymphocytes to viral antigens destroy hepatocytes in mice infected with E1-deleted recombinant adenoviruses. Immunity 1994; 1: 433-442.

6 Yang Y, Su Q, Wilson JM. Role of viral antigens in destructive cellular immune responses to adenovirus vector-transduced cells in mouse lungs. J Virol 1996; 70: 7209-7212.

7 Harty JT, Tvinnereim AR, White DW. CD8+ T cell effector mechanisms in resistance to infection. Annu Rev Immunol 2000; 18: 275-308.

8 Quinn D, Zajac A, Hioe C, Frelinger J. Virus-specific, CD8+ major histocompatibility complex class I-restricted cytotoxic $\mathrm{T}$ lymphocytes in lymphocytic choriomeningitis virus-infected beta2-microglobulin-deficient mice. J Virol 1997; 71: 8392-8396.

9 Worgall S, Wolff G, Falck-Pedersen E, Crystal RG. Innate immune mechanisms dominate elimination of adenoviral vectors following in vivo administration. Hum Gene Ther 1997; 8: $37-44$

10 Schiedner $G$ et al. A hemodynamic response to intravenous adenovirus vector particles is caused by systemic Kupffer cellmediated activation of endothelial cells. Hum Gene Ther 2003; 14: 1631-1641.

11 Muruve DA et al. Helper-dependent adenovirus vectors elicit intact innate but attenuated adaptive host immune responses in vivo. J Virol 2004; 78: 5966-5972.

12 Shayakhmetov DM, Li Z-Y, Ni S, Lieber A. Analysis of adenovirus sequestration in the liver, transduction of hepatic cells, and innate toxicity after injection of fiber-modified vectors. J Virol 2004; 78: 5368-5381.

13 Zhang HG et al. Hepatic DR5 induces apoptosis and limits adenovirus gene therapy product expression in the liver. $J$ Virol 2002; 76: 5692-5700.

14 Kim M, Kim K. Diversity and complexity of CD8+ T cell responses against a single epitope of adenovirus E1B. Virology 2002; 295: 238-249.

15 Toes RE et al. Efficient tumor eradication by adoptively transferred cytotoxic T-cell clones in allogeneic hosts. Int $J$ Cancer 1996; 66: 686-691.

16 Toes RE et al. An adenovirus type 5 early region 1B-encoded CTL epitope-mediating tumor eradication by CTL clones is downmodulated by an activated ras oncogene. J Immunol 1995; 154: 3396-3405.

17 Kaech SM et al. Selective expression of the interleukin 7 receptor identifies effector CD8 T cells that give rise to long-lived memory cells. Nat Immunol 2003; 4: 1191-1198.

18 Yang $\mathrm{Y}$ et al. Cellular immunity to viral antigens limits E1deleted adenoviruses for gene therapy. Proc Natl Acad Sci USA 1994; 91: 4407-4411.

19 Liu ZX, Govindarajan S, Okamoto S, Dennert G. Fas- and tumor necrosis factor receptor 1-dependent but not perforin-dependent pathways cause injury in livers infected with an adenovirus construct in mice. Hepatology 2000; 31: 665-673.

20 Liu Z-X, Govindarajan S, Okamoto S, Dennert G. Fas-mediated apoptosis causes elimination of virus-specific cytotoxic $\mathrm{T}$ cells in the virus-infected liver. J Immunol 2001; 166: 3035-3041.

21 Kafrouni MI, Brown GR, Thiele DL. Virally infected hepatocytes are resistant to perforin-dependent CTL effector mechanisms. J Immunol 2001; 167: 1566-1574.

22 Kafrouni MI, Brown GR, Thiele DL. The role of TNF-TNFR2 interactions in generation of CTL responses and clearance of hepatic adenovirus infection. J Leukoc Biol 2003; 74: 564-571.

23 Altman JD et al. Phenotypic analysis of antigen-specific T lymphocytes. Science 1996; 274: 94-96.

24 Serbina N, Pamer EG. Quantitative studies of CD8+ T-cell responses during microbial infection. Curr Opin Immunol 2003; 15: $436-442$

25 Goldrath AW et al. Cytokine Requirements for acute and basal homeostatic proliferation of naive and memory CD8+ T cells. J Exp Med 2002; 195: 1515-1522.

26 Schluns KS, Kieper WC, Jameson SC, Lefrancois L. Interleukin-7 mediates the homeostasis of naive and memory CD8 T cells in vivo. Nat Immunol 2000; 1: 426-432.

27 Peschon JJ et al. Early lymphocyte expansion is severely impaired in interleukin 7 receptor-deficient mice. J Exp Med 1994; 180: 1955-1960.

28 Wherry EJ et al. Lineage relationship and protective immunity of memory CD8 T cell subsets. Nat Immunol 2003; 4: 225-234.

29 Lusky $\mathrm{M}$ et al. In vitro and in vivo biology of recombinant adenovirus vectors with E1, E1/E2A, or E1/E4 deleted. J Virol 1998; 72: 2022-2032.

30 Worgall $\mathrm{S}$ et al. Role of alveolar macrophages in rapid elimination of adenovirus vectors administered to the epithelial surface of the respiratory tract. Hum Gene Ther 1997; 8: 1675-1684.

31 Wadsworth S, Zhou H, Smith A, Kaplan J. Adenovirus vectorinfected cells can escape adenovirus antigen-specific cytotoxic T-lymphocyte killing in vivo. J Virol 1997; 71: 5189-5196.

32 Belz GT, Altman JD, Doherty PC. Characteristics of virus-specific $\mathrm{CD} 8+\mathrm{T}$ cells in the liver during the control and resolution phases of influenza pneumonia. Proc Natl Acad Sci USA 1998; 95: 13812-13817.

33 Wong P, Pamer EG. Feedback regulation of pathogen-specific T cell priming. Immunity 2003; 18: 499-511.

34 Matsuki $\mathrm{Y}$ et al. Soluble Fas gene therapy protects against Fas-mediated apoptosis of hepatocytes but not the lethal effects 
of Fas-induced TNF-alpha production by Kupffer cells. Cell Death Differ 2002; 9: 626-635.

35 Gross D-A et al. CD4+CD25+ regulatory T cells inhibit immunemediated transgene rejection. Blood 2003; 102: 4326-4328.

36 Sung RS, Qin L, Bromberg JS. TNF(alpha) and IFN(gamma) induced by innate anti-adenoviral immune responses inhibit adenovirus-mediated transgene expression. Mol Therapy 2001; 3 : 757-767.

37 Mueller SN et al. Rapid cytotoxic T lymphocyte activation occurs in the draining lymph nodes after cutaneous Herpes Simplex Virus infection as a result of early antigen presentation and not the presence of virus. J Exp Med 2002; 195: 651-656.

38 Kagi $\mathrm{D}$ et al. Cytotoxicity mediated by $\mathrm{T}$ cells and natural killer cells is greatly impaired in perforin-deficient mice. Nature 1994; 369: 31-37.

39 Zajac AJ, Dye JM, Quinn DG. Control of lymphocytic choriomeningitis virus infection in granzyme B deficient mice. Virology 2003; 305: 1-9.
40 Khakoo SI et al. HLA and NK cell inhibitory receptor genes in resolving hepatitis C virus infection. Science 2004; 305: 872-874.

41 Vogels $\mathrm{R}$ et al. Replication-deficient human adenovirus type 35 vectors for gene transfer and vaccination: efficient human cell infection and bypass of preexisting adenovirus immunity. J Virol 2003; 77: 8263-8271.

42 BIMAS http://bimas.dcrt.nih.gov/molbio/hla_bind/.

43 SYFPEITHI http://syfpeithi.bmi-heidelberg.com/.

44 Olive $\mathrm{M}$ et al. The adenovirus capsid protein hexon contains a highly conserved human CD4+ T-cell epitope. Hum Gene Ther 2002; 13: 1167-1178.

45 Zhang HG et al. Induction of specific T-cell tolerance by adenovirus-transfected, Fas ligand-producing antigen presenting cells. Nat Biotechnol 1998; 16: 1045-1049.

46 Fuller MJ, Zajac AJ. Ablation of CD8 and CD4 T cell responses by high viral loads. J Immunol 2003; 170: 477-486.

47 Khanolkar A, Fuller MJ, Zajac AJ. CD4 T cell-dependent CD8 T cell maturation. J Immunol 2004; 172: 2834-2844. 